\title{
Morality is in the eye of the beholder: the neurocognitive basis of the "anomalous-is-bad" stereotype
}

\author{
Clifford I. Workman, ${ }^{1,2,3}$ (D) Stacey Humphries, ${ }^{1,2,3}$ (D) Franziska Hartung, ${ }^{1,2,3}$ (D) \\ Geoffrey K. Aguirre, ${ }^{1}$ (D) Joseph W. Kable, ${ }^{2,3,4}$ (iD) and Anjan Chatterjee ${ }^{1,2,3}$ (iD \\ ${ }^{1}$ Department of Neurology, University of Pennsylvania, Philadelphia, Pennsylvania. ${ }^{2}$ Penn Brain Science Center, University of \\ Pennsylvania, Philadelphia, Pennsylvania. ${ }^{3}$ Penn Center for Neuroaesthetics, University of Pennsylvania, Philadelphia, \\ Pennsylvania. ${ }^{4}$ Department of Psychology, University of Pennsylvania, Philadelphia, Pennsylvania
}

Address for correspondence: Clifford I. Workman, Ph.D., Department of Neurology, University of Pennsylvania, 3710 Hamilton Walk, Goddard Laboratories, Room 332, Philadelphia, PA 19104. cliffworkmanphd@gmail.com

Are people with flawed faces regarded as having flawed moral characters? An "anomalous-is-bad" stereotype is hypothesized to facilitate negative biases against people with facial anomalies (e.g., scars), but whether and how these biases affect behavior and brain functioning remain open questions. We examined responses to anomalous faces in the brain (using a visual oddball paradigm), behavior (in economic games), and attitudes. At the level of the brain, the amygdala demonstrated a specific neural response to anomalous faces-sensitive to disgust and a lack of beauty but independent of responses to salience or arousal. At the level of behavior, people with anomalous faces were subjected to less prosociality from participants highest in socioeconomic status. At the level of attitudes, we replicated previously reported negative character evaluations made about individuals with facial anomalies, and further identified explicit biases directed against them as a group. Across these levels of organization, the specific amygdala response to facial anomalies correlated with stronger just-world beliefs (i.e., people get what they deserve), less dispositional empathic concern, and less prosociality toward people with facial anomalies. Characterizing the "anomalous-is-bad" stereotype at multiple levels of organization can reveal underappreciated psychological burdens shouldered by people who look different.

Keywords: amygdala; faces; disgust; empathy; morality; neuroaesthetics

\section{Introduction}

Hollywood villains are more likely than heroes to have facial anomalies (e.g., scars). ${ }^{1}$ Harry Potter, for instance, inspired fear in a reptilian Voldemort, A Nightmare on Elm Street scared us awake with Freddy Krueger's marred visage, and The Lion King taught us never to trust a Scar. Why should facial anomalies bear on our moral attitudes? For many people, their facial anomalies render them unjust targets of discrimination. ${ }^{2-6}$

People expect more positive character traits in those with attractive faces, an effect dubbed the "beauty-is-good" stereotype. ${ }^{7,8}$ Cues of facial beauty (e.g., symmetry) predict positive health outcomes, suggesting preferences for beauty are an adaptive way to assess quality in potential romantic partners. ${ }^{9}$ Judgments of facial attractiveness and trustworthiness reportedly share a common neural substrate in the amygdala, lending neurobiological plausibility to the interrelatedness of beauty and morality. ${ }^{10}$ Evolutionary pressures, however, may have actually favored an "unattractive-is-bad" stereotype. ${ }^{11}$ On this view, although face perception generally extracts adaptive information, ${ }^{12}$ otherwise adaptive cues may generalize beyond features linked to evolutionary fitness-facial anomalies, for instance, may signal poor health even when unrelated to illness. 
An "anomalous-is-bad" stereotype is hypothesized to facilitate biases against people with anomalous faces. Three lines of recent evidence support this hypothesis: First, people made harsher character judgments about individuals with facial anomalies before versus after surgical correction. ${ }^{4}$ Second, people demonstrated robust implicit biases against facial anomalies. ${ }^{2}$ Third, using fMRI, anomalous faces elicited less activation in dorsal anterior cingulate cortex (dACC $)^{2}$ before versus after surgical correction. This pattern of blunted dACC response has been proposed as a neural biomarker for "dehumanization." ${ }^{3}$ Dehumanization is "perceiving a person or group as lacking humanness." 14 This tautology underscores the theoretical difficulty in conceptualizing "humanness." 15 One view allows that dehumanized others may appear human despite having subhuman essences. Alternatively, dehumanized others may retain their essences while lacking distinctly human psychological capacities. Regardless of mechanism, dehumanization manifests in moral behavior as diminished prosociality and/or elevated antisociality. ${ }^{14}$ It is not yet known whether the "anomalousis-bad" stereotype facilitates dehumanizing behavior.

Moral behavior is shaped by moral emotions (e.g., disgust and empathy) and values (e.g., justice and egalitarianism). ${ }^{16,17}$ Facial anomalies reportedly elicit disgust responses that indicate they are regarded as expressions of infectious disease. ${ }^{18,19}$ This is consistent with models suggesting disgust evolved to facilitate pathogen avoidance, ${ }^{20}$ pointing toward both distal evolutionary (i.e., pathogenaversion) and proximate psychological causes (i.e., disgust) of the "anomalous-is-bad" stereotype. Effects of disgust on moral judgment are small ${ }^{21}$ and may not completely capture the proximate cause of the stereotype, however, which could instead emerge from diminished prosocial emotions (i.e., empathic concern) and/or from social values. Furthermore, instead of dehumanization, dACC blunting may track beauty since people with facial anomalies are generally regarded as less attractive (we do not, however, wish to equate anomalies with ugliness-anybody, regardless of beauty, can acquire facial anomalies). Alternatively, facial anomalies may be particularly salient, or they may arouse especially strong emotions in viewers.
In this research, we first used an online survey to characterize attitudes toward people with facial anomalies. In a second study, we probed neural responses to anomalous and typical faces (Fig. 1) using an fMRI implementation of the classic oddball paradigm. ${ }^{22}$ During scanning, participants viewed a familiarized set of average-looking faces interspersed with novel faces. The novel faces had visible anomalies or were nonanomalous (either average-looking or attractive). This research leveraged the design of the oddball task, together with measures of attitudes, behaviors, and dispositions, to address the following hypotheses (Fig. 2):

1. An "anomalous-is-bad" stereotype is expressed in negative attitudes about people with facial anomalies. Participants were predicted to make harsher character inferences in response to anomalous (relative to typical) faces and to display implicit biases against anomalous faces. ${ }^{2,4}$

2. The stereotype facilitates the mistreatment of people with facial anomalies. Compared with typical faces, facial anomalies were predicted to elicit more antisociality and less prosociality in behavioral economic games. Economic behaviors-whether prosocial (i.e., donating money to others) or antisocial (i.e., withholding money from others) - are contingent on individual economic prosperity. ${ }^{23,24}$ In other words, the amount of money someone is willing to sacrifice to benefit or harm others depends on how much money they have to begin with. To account for individual differences in economic standing, we included socioeconomic status (SES) in our behavioral models.

3. The dACC responds to emotionally arousing faces. This hypothesis predicts that anomalous and attractive (relative to average)-looking faces will implicate the dACC. ${ }^{2}$

4. Information represented on some levels of organization (i.e., attitudes and dispositions) is also represented on others (i.e., brain activations and behavior). The neural response specific for facial anomalies, for instance, is predicted to track implicit biases against them. Such neural responses are also predicted to correlate with sensitivity to pathogen-related disgust. 


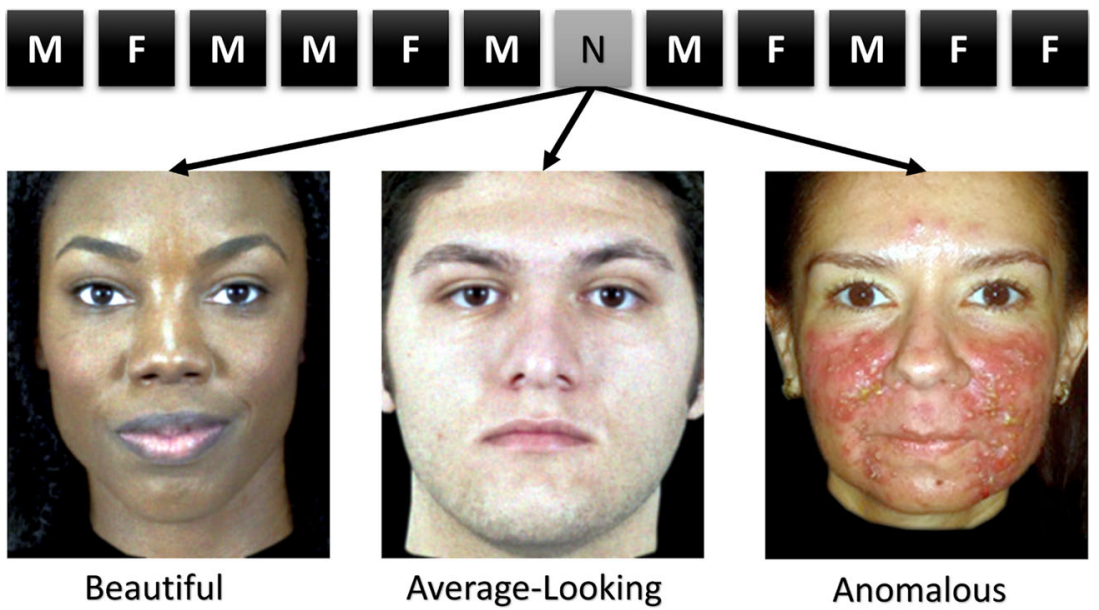

Figure 1. Schematic of the oddball fMRI task design. Participants completed 540 trials of an oddball task ( 5 repetitions each of 90 learned faces ( 450 total), 1 instance each of 90 novel faces ( 30 anomalous, 30 beautiful, and 30 average-looking)). Trials started with a photograph of a male or female face for $500 \mathrm{~ms}$, followed by a null event for $1000 \mathrm{~ms}$ plus jitter lasting an average of 1833 ms (3333 ms per trial). F, female; M, male; N, novel.

\section{Materials and methods}

\section{Participants}

For the online survey study, a sample of $n=451$ participants was recruited via Amazon's Mechanical Turk service to complete a survey delivered online through the Qualtrics platform (178 females; age: $35.53 \pm 10.47$ years; education: $14.85 \pm 2.40$ years). Participants were compensated for their time. Using effect sizes calculated from the data reported by Jamrozik and colleagues, ${ }^{4}$ a minimum of 102 responses per dimension was expected to provide sufficient power $(80 \%)$ to detect differences in character judgments between anomalous and typical faces (Table S1, online only), and also to be sufficient to reliably estimate face ratings (Cronbach's $\alpha>0.8$ ). We targeted 120 responses to each of the face-rating dimensions to provide a buffer against exclusions of low-quality data. From the original $n=451$ participants, data from 29 participants were excluded for failing more than two out of five attention checks. Data from one participant were excluded because they acknowledged that their responses were of poor quality. ${ }^{25}$ Finally, data were excluded for 18 participants who chose not to report their sex and/or sexual orientation, both of which were expected to interact with ratings of facial attractiveness. The final sample consisted of $n=403$ participants (168 females; age: 35.69 \pm 10.50 years (range: 19-72); education: 15.03 \pm 2.19 years (range: 4-25); race/ethnicity: 293 white, 38 black, 21 Asian, 26 Hispanic or Latinx, 2 American-Indian/Alaskan-Native, 21 multiracial, 1 "other," and 1 nonresponse; sexual orientation: 362 heterosexual, 15 homosexual, and 26 bisexual).

A second independent sample of $n=32$ participants was recruited for the fMRI study (13 females; age: $26.75 \pm 8.65$ years; education: $16.34 \pm 2.74$ years). This sample size was chosen to maximize power to detect large effects, given that notable improvements in power have been reported after increasing sample sizes from $\mathrm{N}=20$ to $\mathrm{N}=30-40$ participants. $^{26,27}$ Participants were recruited from the Philadelphia metropolitan area using online advertisements and they received monetary compensation for their time. The inclusion criteria were: 18 years of age or older, no contraindications for MRI scanning (e.g., claustrophobia), no use of psychotropic medications, and no previous head traumas resulting in losses of consciousness for $15 \mathrm{~min}$ or longer. Two participants completed all study procedures save for the MRI (one was excluded for actively using psychotropic medications and another for having metal in their body that could not be removed before scanning). MRI data for two participants were excluded because of excessive inscanner head motion in one participant (the criteria are elaborated below) and significant morphological abnormalities in the other. The final sample consisted of $n=27$ participants ( 13 females; age: 25.52 


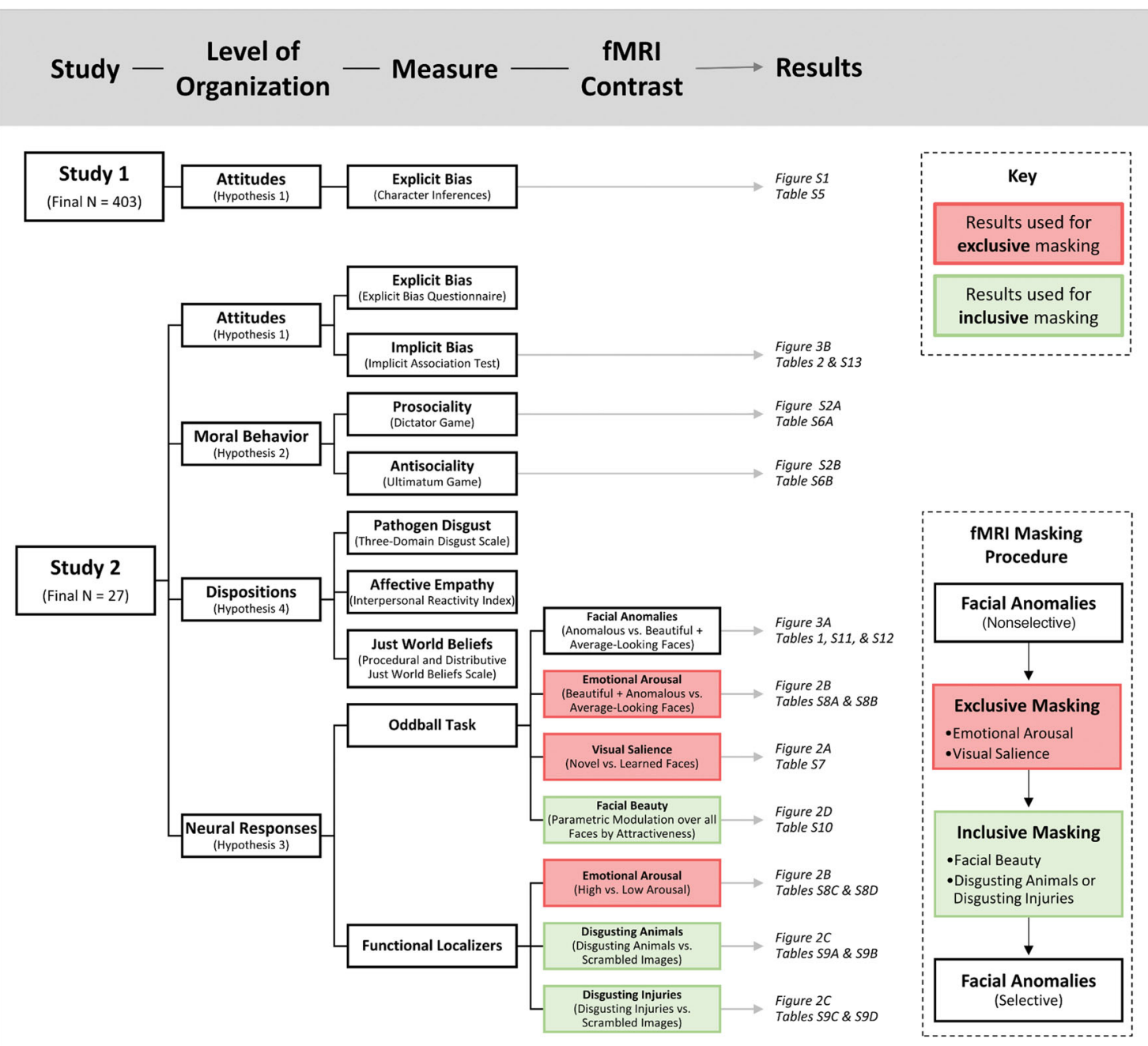

Figure 2. A visual overview of the research design. This overview delineates between the two studies reported here; the levels of organization they investigated; the measures that were examined, including fMRI contrasts; and where the corresponding resultsfigures and tables, specifically-are located. The inset panel contains an overview of the fMRI masking procedure. Red boxes distinguish fMRI analyses used for exclusive masking, whereas green boxes signify inclusive masking.

\pm 7.12 years (range: $18-46$ ); education: $16.41+$ 2.65 years (range: $12-23$ ); race/ethnicity: 11 white, 6 black, 6 Asian, 2 Hispanic or Latinx, and 2 multiracial; sexual orientation: 21 heterosexual, 2 homosexual, 1 bisexual, 1 "other," and 2 nonresponses).

\section{Stimuli}

In both studies, photographs of people with facial anomalies were drawn from the ChatLab Facial Anomaly Database (e.g., Fig. 1). ${ }^{28}$ Identified with the Face Image Meta-Database search engine (https://fimdb.clffwrkmn.net/), we also chose the
Chicago Face Database for images of people without facial anomalies. ${ }^{29}$ The stimuli selected from these databases were well matched on sex and race/ethnicity, although people with facial anomalies were generally older than those with typical faces (see Table S3, online only).

\section{Online survey study}

The online survey was adapted from Jamrozik and colleagues, ${ }^{4}$ with eight questions about emotional reactions to and perceptions of people in photographs. The questions were selected to 
ensure the coverage of all four significant principal components described previously. ${ }^{4}$ Limiting the number of questions reduced the burden placed on participants-with each participant rating 50 (of 180) faces, and 8 questions per face (400 questions total), the survey usually took over $30 \mathrm{~min}$ to complete. Before starting the survey, participants were instructed they would first rate each photograph according to "how the face made you feel." Next, they were told they would rate their impressions of the person in the photograph. After a practice trial, participants started the survey.

As in our previous study, each photograph was presented for about $2.5 \mathrm{~s}$ before participants were redirected to a separate page to provide their ratings. The nine-point pictorial Self-Assessment Manikin (SAM) scale measured emotional reactions to the stimuli in terms of valence, arousal, and dominance. ${ }^{30}$ Five-point semantic differential scales examined perceptions of the people in the photographs in terms of personality characteristics (e.g., anxious), internal attributes (e.g., contentedness), social traits (e.g., confidence, dominance, and trustworthiness), and attractiveness. Linear mixed effects models (LMEMs) in RStudio examined whether attitudinal manifestations of the "anomalous-is-bad" stereotype were replicated when comparing anomalous with typical faces with no known history of visible difference (average looking and beautiful). We expected anomalous faces to elicit worse ratings than typical faces on attractiveness, trustworthiness, contentedness, dominance, anxiousness, and perceiver arousal, dominance, and happiness. ${ }^{4}$

\section{fMRI study}

Dispositions. Participants completed a Just World Beliefs Scale, ${ }^{31}$ which measures beliefs about interpersonal fairness toward oneself and others; the Interpersonal Reactivity Index, ${ }^{32}$ which measures cognitive (perspective taking) and affective (empathic concern) empathy; and a subscale from the Three-Domain Disgust scale ${ }^{33}$ that measures sensitivity to pathogen-related disgust.

Attitudes. The Explicit Bias Questionnaire $(\mathrm{EBQ})^{2}$ was used to quantify explicit biases against people with facial anomalies overall (rather than against individual faces; Table S2, online only). The Implicit Association Test (IAT) ${ }^{2}$ - used extensively to measure implicit biases against social out- groups $^{34,35}$-was used to quantify implicit biases against people with facial anomalies. One-tailed nonparametric Wilcoxon signed rank tests were used to detect explicit and implicit biases against anomalous faces.

Prosociality. In the Dictator Game (DG), one player (the dictator) decides how to split an endowment (\$5) with another player (the receiver). ${ }^{36}$ Participants played 15 rounds as the dictator. Photographs ostensibly depicting the receiver showed people with facial anomalies before (7-8 rounds) or after (7-8 rounds) corrective surgery. In each trial, participants chose between two randomly selected splits, ranging from keeping the entire $\$ 5$ to sharing it 50:50. The split entailing the smallest difference in payoffs between dictator and receiver was considered prosocial. A model was constructed that regressed prosociality against face type (Anomalous Face Pre-Surgery | Post-Surgery), difference between payoffs, and SES, with random intercepts for subject and face stimulus.

Antisociality. The Ultimatum Game (UG) is similar to the DG, except receivers can reject offers so that neither player receives any part of the endowment. ${ }^{37}$ Playing as receiver for 60 rounds, participants saw a photograph of a person with or without a facial anomaly before being shown an offer ostensibly proposed by that person. Participants in fact played both games with computergenerated partners. Offers ranged from (receiver: proposer) $\$ 4.50$ : $\$ 0.50$ to $\$ 0.00$ : $\$ 5.00$. A binary variable indexed antisociality, reflected in decisions to reject offers. A binomial LMEM regressed antisociality on face type (Anomalous | Beautiful), payoff difference, and SES, with random intercepts for subject and face stimulus. Although UG rejections are often interpreted as "altruistic punishment" — costly punishment to discourage future exploitation-we sought to isolate antisociality by contrasting rejections of proposers with and without anomalies.

fMRI tasks. Before scanning, participants completed a 1-back working memory task to learn a set of photographs depicting typical faces of average attractiveness (Table S2, online only) without known histories of visible anomalies. During scanning, participants completed 540 trials of an oddball task over five runs (Fig. 1; 5 repetitions of 90 learned faces (450 total), 1 instance each of 90 
novel faces (30 anomalous, 30 beautiful, and 30 average-looking); $16.7 \%$ novel). Face photographs (500 ms) were followed by a null event $(1000 \mathrm{~ms})$ plus jitter ( $\sim 1833$ milliseconds). Similar parameters were used previously. ${ }^{38,39}$ The ordering and spacing of trials was optimized with optseq2 (https://surfer. nmr.mgh.harvard.edu/fswiki/optseq2). Participants counted the number of novel faces in each run. ${ }^{40}$ Additional functional scans were acquired while participants passively viewed images to localize neural responses to disgusting animals and bodily injuries ${ }^{41}$ and emotional arousal..$^{42}$ Each localizer comprised a single block of 16 trials that alternated between subsets of 8 images from the target category and 8 from a control condition (scrambled versions of the disgusting images; low arousal images).

MRI acquisition and preprocessing. MRI data were acquired on a 3T Siemens Magnetom Prisma (Erlangen, Germany) with a 64-channel head coil using Human Connectome Project sequences: gradient-echo echo-planar images (EPIs; 520 volumes per run; 72 axial slices; $2 \mathrm{~mm}$ slice thickness; interleaved acquisition; repetition time: $720 \mathrm{~ms}$; echo time: $37 \mathrm{~ms}$; multiband factor: 8; field of view: $208 \times 144 \mathrm{~mm}$; reconstructed voxel size: 2 $\mathrm{mm}^{3}$; flip angle: $52^{\circ}$ ) and T1-weighted anatomical images. Preprocessing was performed in SPM12 (https://www.fil.ion.ucl.ac.uk/spm/). The EPIs were subjected to frame-by-frame realignment to correct for head motion before high-pass filtering (128 s cutoff). Realignment generated motion parameters used as nuisance regressors in first-level models, and mean EPIs to which corresponding anatomical images were coregistered. After coregistration, anatomical images were segmented to generate nonlinear warping parameters. Once warped into standard space, EPIs were smoothed with a 4-mm Gaussian kernel. Volumes with excessive head motion ( $>0.5 \mathrm{~mm}$ per TR) were interpolated and deweighted in first-level models (https://cibsr.stanford.edu/tools/humanbrain-project/artrepair-software.html). First-level contrasts tested the predictions described above. An additional set of first-level models included mean-centered parametric modulators for average attractiveness ratings derived from the online survey study. First-level models were also constructed for each of the functional localizers.
Neuroimaging analysis. For the oddball fMRI task, contrasts at the second level captured neural responses associated with visual salience and emotional arousal. For the functional localizers, second-level models identified brain areas implicated in processing disgusting and arousing images. The resulting statistical maps were combined, binarized, and used to mask later analyses. Beyond simply excluding nonselective effects that may be attributable to salience or arousal, we also sought out areas implicated in processing both morality (i.e., disgust) and beauty (i.e., facial unattractiveness). A mask representing group-level parametric variation in neural responses to facial attractiveness was binarized and combined with masks for disgust toward animals and toward injuries. It is important to note that masking, in contrast to methods like small volume correction, does not alter significance but only the inclusion and/or exclusion of voxels.

Voxel-wise analyses used an uncorrected cluster-forming threshold of $\alpha=0.001$ and an extent threshold of $\mathrm{k}=13$ voxels (determined with nonparametric Monte Carlo simulations; https://afni.nimh.nih.gov/pub/dist/doc/ program_help/3dClustSim.html). Results were significant at a cluster-level FWE-corrected $\alpha=0.01$. Regions of interest (ROIs; 6-mm spheres) were centered at coordinates chosen a priori (dACC and lateral occipital cortices (LOC) $)^{2}$ or defined functionally from Table 1 (fusiform and amygdala): dACC $(\mathrm{x}= \pm 2, \mathrm{y}=36, \mathrm{z}=10)$, LOC (left: $-28,-98,8$; right: $34,-90,2$ ), fusiform (left: $-32,-60,-13$; right: $33,-57,13)$, and amygdala $(-25,1,-19)$. Percent signal change was extracted from each ROI with MarsBaR (http://marsbar.sourceforge.net/). Relations between ROI and other measures were assessed with nonparametric correlations (uncorrected for multiple comparisons).

\section{Results}

\section{Hypothesis 1-Attitudes}

People with facial anomalies were rated less trustworthy than beautiful faces, and less attractive, less content, and more anxious than people with both beautiful and average-looking faces (Table S5, online only; Fig. S1, online only; see also the Supplementary Results, online only). Raters felt more aroused by anomalous compared with averagelooking but not beautiful faces, and less happy when looking at anomalous (relative to typical) 
Table 1. Neural responses selective for faces with visible anomalies

A: Anomalous $>$ (beautiful + average-looking novel faces)

Masking: inclusive of disgusting animals and facial beauty; exclusive of visual salience and emotional arousal

\begin{tabular}{|c|c|c|c|c|c|}
\hline \multirow[b]{2}{*}{ Brain regions by cluster } & \multirow[b]{2}{*}{ Cluster size } & \multicolumn{3}{|c|}{ Peak MNI coordinates } & \multirow[b]{2}{*}{ Peak $t$ statistic } \\
\hline & & $x$ & $y$ & $z$ & \\
\hline L fusiform & 92 & -35 & -62 & -13 & 8.16 \\
\hline L amygdala & 13 & -25 & 1 & -19 & 7.01 \\
\hline R fusiform & 22 & 33 & -57 & -13 & 6.11 \\
\hline \multicolumn{6}{|c|}{ B: $($ Beautiful + average-looking novel $)>$ anomalous faces } \\
\hline
\end{tabular}

L, left; MNI, Montreal Neurological Institute; NS, nonsignificant; R, right.

faces. Feelings of dominance in the perceiver or face were not seen, which is a result that did not replicate earlier findings. ${ }^{4}$ Explicit bias scores on the EBQ were elevated (median $=0.071, \mathrm{~V}(32)=335$, $r=0.296, P=0.047,95 \%$ CI: 0.03-0.60). Elevated scores on the IAT indicated slight bias but did not reach significance (median $=0.200, \mathrm{~V}(31)=318$, $r=0.246, P=0.088,95 \%$ CI: $0.01-0.54)$.

\section{Hypothesis 2-Behavior}

For prosocial behavior assessed with the Dictator Game, there was a significant interaction between face type, SES, and difference between payoffs $(\beta=-1.002$, SE $=0.464, z=-2.158, P=0.031$; see Table S6 (online only) for remaining fixed effects). Participants with the highest SES-that is, those most likely to have expendable income-were the least inclined to act prosocially toward people with facial anomalies (Fig. S2A, online only). For antisocial behavior assessed with the Ultimatum Game, an interaction between face type (Anomalous $\mid$ Beautiful) and SES approached but did not reach significance $(\beta=1.547, \mathrm{SE}=0.818, z=1.892$, $P=0.059$; Fig. S2B, online only).

\section{Hypothesis 3-Brain functioning}

Neural sensitivity to visual salience. Relative to faces learned outside the scanner, appearances of novel faces elicited stronger activations in the bilateral anterior insula, bilateral supplementary motor area/dACC, bilateral ventral striatum (VS), bilateral fusiform gyri, left thalamus, right hippocampus, bilateral inferior parietal lobule (IPL), bilateral dorsolateral prefrontal cortex (dlPFC), left lateral orbitofrontal cortex (latOFC), left inferior frontal gyrus (IFG), right posterior cingulate cortex (PCC), midbrain, and cerebellum (Table S7A, online only; Fig. 3A).

Learned faces, by contrast, elicited stronger activation in the left hippocampus, bilateral PCC, right precuneus, left ventromedial prefrontal cortex, bilateral VS (nonoverlapping with the bilateral VS cluster implicated by the opposite contrast), bilateral middle frontal gyri, right precentral gyrus, right superior temporal gyrus (STG), right posterior insula, left middle temporal gyrus (MTG), and right caudate (Table S7B, online only; Fig. 3A).

\section{Neural sensitivity to emotional arousal.}

Stronger activation was observed for highly arousing faces (novel beautiful and anomalous) relative to faces low in arousal (novel average-looking) in a single cluster in the right fusiform gyrus (Table S8A, online only; Fig. 3B). Average-looking faces did not elicit stronger activations than beautiful and anomalous faces in any regions. In addition to the oddball task, a functional localizer task identified neural responses sensitive to emotional arousal. Relative to images low in emotional arousal (e.g., a light bulb), highly arousing negative imagery (e.g., a spider) resulted in stronger neural responses in the bilateral fusiform, left IFG, and bilateral precentral gyri (Table S8C, online only; Fig. 3B), and weaker responses elsewhere in the bilateral fusiform and in the left PCC (Table S8D, online only; Fig. 3B).

Neural sensitivity to disgust. During two blocks of the functional localizer task, participants viewed images rated high on disgust, along with scrambled versions of those same images. Given that neural responses to disgusting images were compared with scrambled versions of those same images, the 

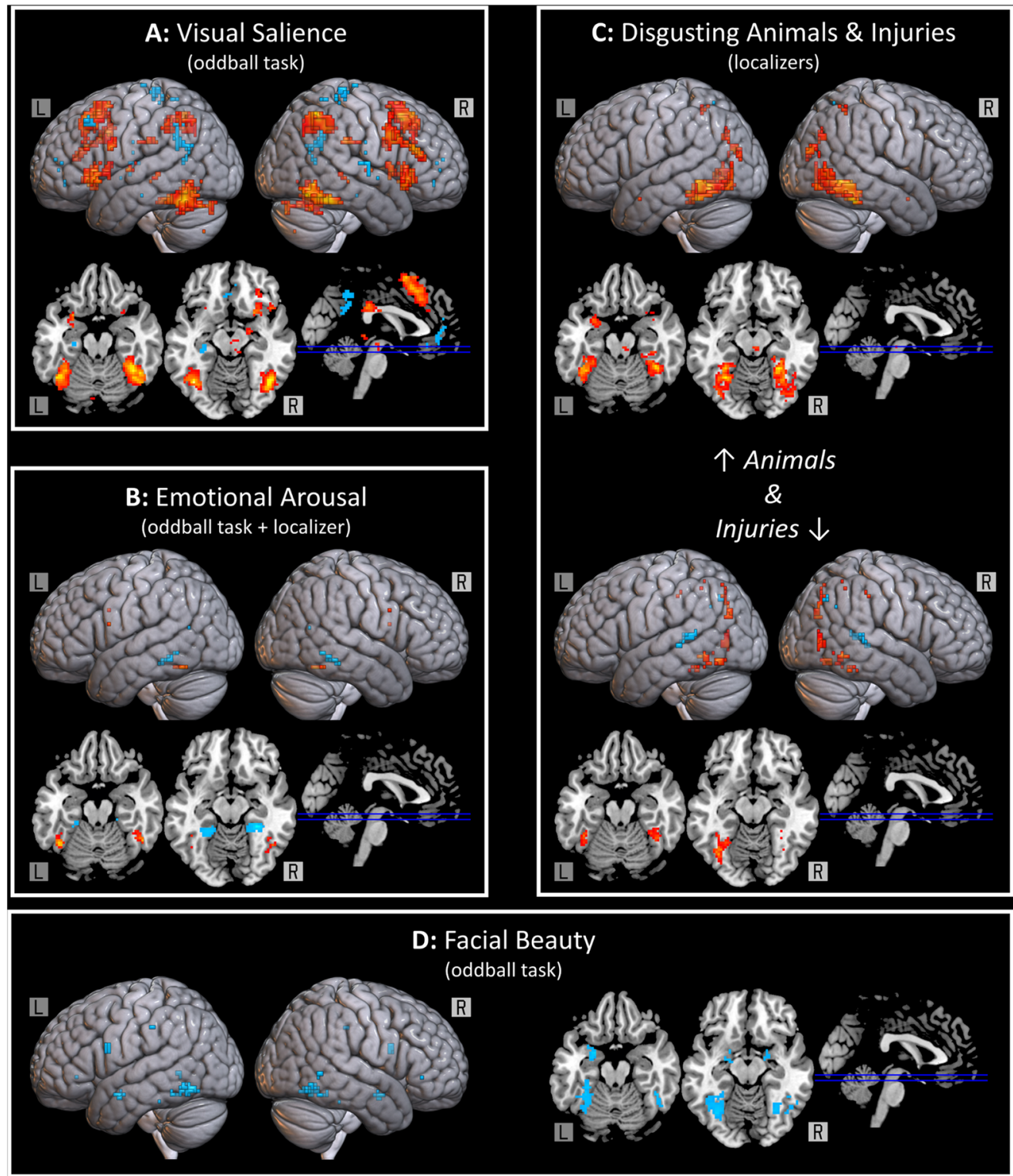

D: Facial Beauty (oddball task)

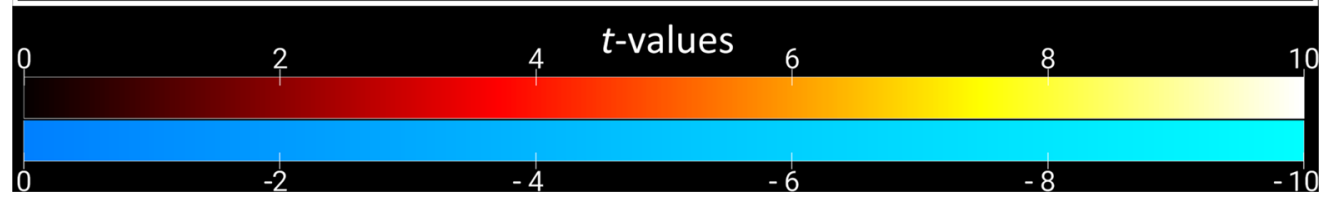

Figure 3. Brain regions implicated by the oddball and functional localizer tasks. (A) Neural sensitivity to the salience of faces was characterized with the oddball task. Warm colors reflect increased activation for visually salient faces. Cool colors reflect decreased activation. (B) The neural response to arousing images of faces (beautiful and anomalous) was compared against responses to average-looking faces low in arousal. This is shown together with the functional localizer for arousal. Warm colors reflect increased activation with increasing arousal. Cool colors reflect negative relations to arousal. (C) During the functional localizer, participants saw disgusting images of animals (e.g., leeches and cockroaches; top) and bodily injuries (bottom) along with scrambled versions of those images. Warm colors reflect stronger activation for disgusting versus scrambled images. Cool colors capture the reverse contrast. (D) A parametric modulation analysis characterized relations between hemodynamic response and attractiveness. Cool colors indicate negative relations. 
disgust localizers may have been confounded with simple visual responding (e.g., to objects versus nonobjects). In attempting to characterize selective neural responses to facial anomalies below, this hypothesis is tested by inclusively masking for disgust while exclusively masking for visual saliencethe resulting statistical map includes only areas that were sensitive to disgust and insensitive to simple visual responding. In one block, the images depicted typically disgusting animals, such as leeches and cockroaches, whereas in the other block, the images depicted bodily infections and wounds. Relative to their scrambled counterparts, images of disgusting animals elicited greater activation in the bilateral amygdala, bilateral fusiform gyri, bilateral superior occipital cortex, bilateral superior parietal lobule (SPL), and midbrain (Table S9C, online only; Fig. $3 \mathrm{C}$ ). The reverse contrast identified a single cluster in the precuneus (Table S9B, online only; Fig. 3C). Larger neural responses to disgusting injuries, on the other hand, were observed in the bilateral fusiform, bilateral precuneus, right IPL, right MTG, and left precentral gyrus (Table S9C, online only; Fig. 3C). The reverse contrast identified clusters in the right SPL, left putamen, left MTG, and bilateral STG (Table S9D, online only; Fig. 3C).

Neural sensitivity to facial beauty. No significant positive relations between hemodynamic response amplitudes and average facial beauty ratings were detected. Significant negative relations, however, were observed in the bilateral fusiform gyri, bilateral amygdala, bilateral dlPFC, bilateral intraparietal sulcus (IPS), left temporoparietal junction, left IFG, left thalamus, left MTG, and left SMG (Table S10, online only; Fig. 3D).

Neural sensitivity to facial anomalies. Relative to typical novel faces, anomalous faces increased activation in the bilateral fusiform, bilateral amygdala, right hippocampus, right latOFC, right VS, right superior occipital cortex, left IFG, bilateral IPS, and left IPL (Table S11A, online only). Decreased activation was detected in the bilateral STG and bilateral somatosensory cortices (Table S11B, online only). This analysis was repeated with a mask that included response to disgusting animals and excluded response to visual salience and emotional arousal (Fig. 3). We found increased activation restricted to the bilateral fusiform gyri and left amygdala (Table S12A, online only), and no areas of decreased activation. Repeating this analysis with a mask that included response to disgusting injuries and excluded response to salience and arousal (Fig. 3) revealed increased activation in response to anomalous (relative to typical) faces in the bilateral fusiform gyri (Table S12C, online only), overlapping with the fusiform clusters activated by disgusting animals. Finally, with a mask that included response to facial beauty and excluded response to visual salience and emotional arousal (Fig. 3), we found increased activation in response to anomalous (compared with beautiful and average-looking) novel faces in the bilateral fusiform gyri, bilateral amygdala, right posterior superior temporal sulcus, left IFG, left IPL, and right IPS (Table S12E, online only). Independent of salience or arousal, only the amygdala and fusiform gyri showed specific neural responses to anomalous faces and sensitivity to disgust and unattractiveness (Table 1A and Fig. 4A).

\section{Hypothesis 4-Across levels of organization}

For anomalous (relative to typical) novel faces, we found a positive correlation between IAT $\mathrm{d}^{\prime}$ scores and activation in the bilateral fusiform, bilateral amygdala, right hippocampus, left latOFC, left IFG, left IPL, and bilateral IPS (Table S13A, online only). Negative correlations were found in the left precuneus, left MTG, bilateral STG, and bilateral somatosensory cortices (Table S13B, online only). This analysis was repeated with a mask for responses to facial beauty and disgusting animals that excluded response to visual salience and emotional arousal (Fig. 3). We found positive correlations restricted to the bilateral fusiform gyri and left amygdala (Table 2A and Fig. 4B) and no negative correlations. Of note, the cluster in right fusiform gyrus from Table 1 was negatively associated with implicit biases toward anomalous faces $\left(r_{s}(27)=-0.515, P=0.007,95 \% \mathrm{CI}:-0.752\right.$ to -0.159$)$. Percent signal change in the dACC correlated negatively with dispositional empathic concern $\left(r_{s}(27)=-0.431, P=0.025,95 \% \mathrm{CI}:-0.697\right.$ to 0.061 ), with dACC blunting in response to anomalies occurring in those highest in empathic concern. Percent signal change in the left amygdala (Table 1) related positively to justice beliefs toward others $\left(r_{s}(27)=0.463, P=0.015\right.$, 95\% CI: 0.101-0.717) and negatively to dispositional empathic concern $\left(r_{s}(27)=-0.411, P=0.033,95 \%\right.$ CI: -0.684 to $-0.037)$. The same amygdala cluster demonstrated 
Table 2. Correlations between implicit biases and neural responses selective for facial anomalies

\begin{tabular}{|c|c|c|c|c|c|}
\hline \multicolumn{6}{|c|}{$\begin{array}{c}\text { A: Positive correlation with IAT d' scores, anomalous }>\text { (beautiful + average-looking novel faces) } \\
\text { Masking: inclusive of disgusting animals; exclusive of visual salience and emotional arousal }\end{array}$} \\
\hline & & \multicolumn{3}{|c|}{ Peak MNI coordinates } & \multirow[b]{2}{*}{ Peak $t$ statistic } \\
\hline Brain regions by cluster & Cluster size & $x$ & $y$ & $z$ & \\
\hline L fusiform & 87 & -35 & -62 & -13 & 8.27 \\
\hline $\mathrm{R}$ fusiform & 20 & 33 & -57 & -13 & 7.19 \\
\hline L amygdala & 13 & -25 & 1 & -19 & 6.61 \\
\hline \multicolumn{6}{|c|}{ B: Negative correlation with IAT d' scores, anomalous $>$ (beautiful + average-looking novel faces) } \\
\hline \multicolumn{6}{|c|}{ Masking: inclusive of disgusting animals; exclusive of visual salience and emotional arousal } \\
\hline \multicolumn{6}{|c|}{ NS } \\
\hline
\end{tabular}

L, left; MNI, Montreal Neurological Institute; NS, nonsignificant; R, right.

a negative correlation with the frequency of prosocial decisions toward anomalous $\left(r_{s}(27)=-0.430\right.$, $P=0.025,95 \%$ CI: -0.697 to 0.060$)$ but not typical faces $(P=0.126)$. Similar relations characterized the fusiform (Table 1; left: $r_{s}(27)=-0.383, P=0.048$, $95 \%$ CI: -0.666 to -0.004 ; right: $r_{s}(27)=-0.345$, $P=0.078,95 \%$ CI: -0.621 to 0.074$)$.

\section{Discussion}

Motivated by the well-documented "beauty-isgood" stereotype, ${ }^{7}$ we tested the hypothesis that a complementary "anomalous-is-bad" stereotype expresses itself in attitudes, behavior, brain functioning, and dispositions. Anomalous faces were rated less attractive, less content, and more anxious than typical faces, and less trustworthy than beautiful faces, replicating earlier work. ${ }^{4}$ Participants also felt more aroused and less happy looking at anomalous (relative to typical) faces. Participants expressed explicit biases against people with facial anomalies, but their implicit biases were slight and not significant. Nevertheless, we found evidence for the "anomalous-is-bad" stereotype in explicit negative attitudes about people with facial anomalies both as individuals (i.e., character inferences) and as a group (i.e., scores on the Explicit Bias Questionnaire).

The significance of attitudinal biases against facial anomalies hinges on whether such biases facilitate dehumanizing behavior. We detected a significant interaction between prosociality and individual differences in economic prosperity, such that participants highest in SES-that is, those most likely to have expendable income-gave less to people with anomalous (relative to typical) faces in the DG. Previous research detected positive relations between prosociality and social and financial status. $^{23,24}$ These studies were not designed, however, to characterize potential synergistic effects between SES and intergroup biases. In an extension to this earlier work, we found that effects of SES on prosociality depend on intergroup context. Antisociality assessed with the UG, on the other hand, was not significantly elevated against people with facial anomalies.

We did not detect significant main effects of facial anomalies on prosocial or antisocial behavior. The absence of evidence for such effects, however, is not itself evidence of their absence. It may be that the Dictator and/or Ultimatum Games are poor proxies for the kinds of real-world discriminatory behaviors associated with the "anomalous-is-bad" bias. ${ }^{2-6}$ Another possibility is that our sample was too small to achieve the statistical power needed for adequate sensitivity. A meta-analysis of studies on the consequences of intergroup bias for economic game behavior found wide variation in average effect sizes depending on the specific group-based identity made salient (i.e., $\mathrm{d}=-0.177$ to 0.551 , or from small to medium in magnitude). ${ }^{43}$ Counterintuitively, stronger discrimination in economic games was detected for experimentally contrived groups than for facets of identity like ethnicity and nationality that some participants might not find particularly salient. ${ }^{43}$ It remains to be seen whether increasing the salience of facial anomalies exacerbates intergroup biases as expressed in economic games. Furthermore, while the DG contrasted anomalous faces before and after surgical correction, the UG contrasted anomalous and 


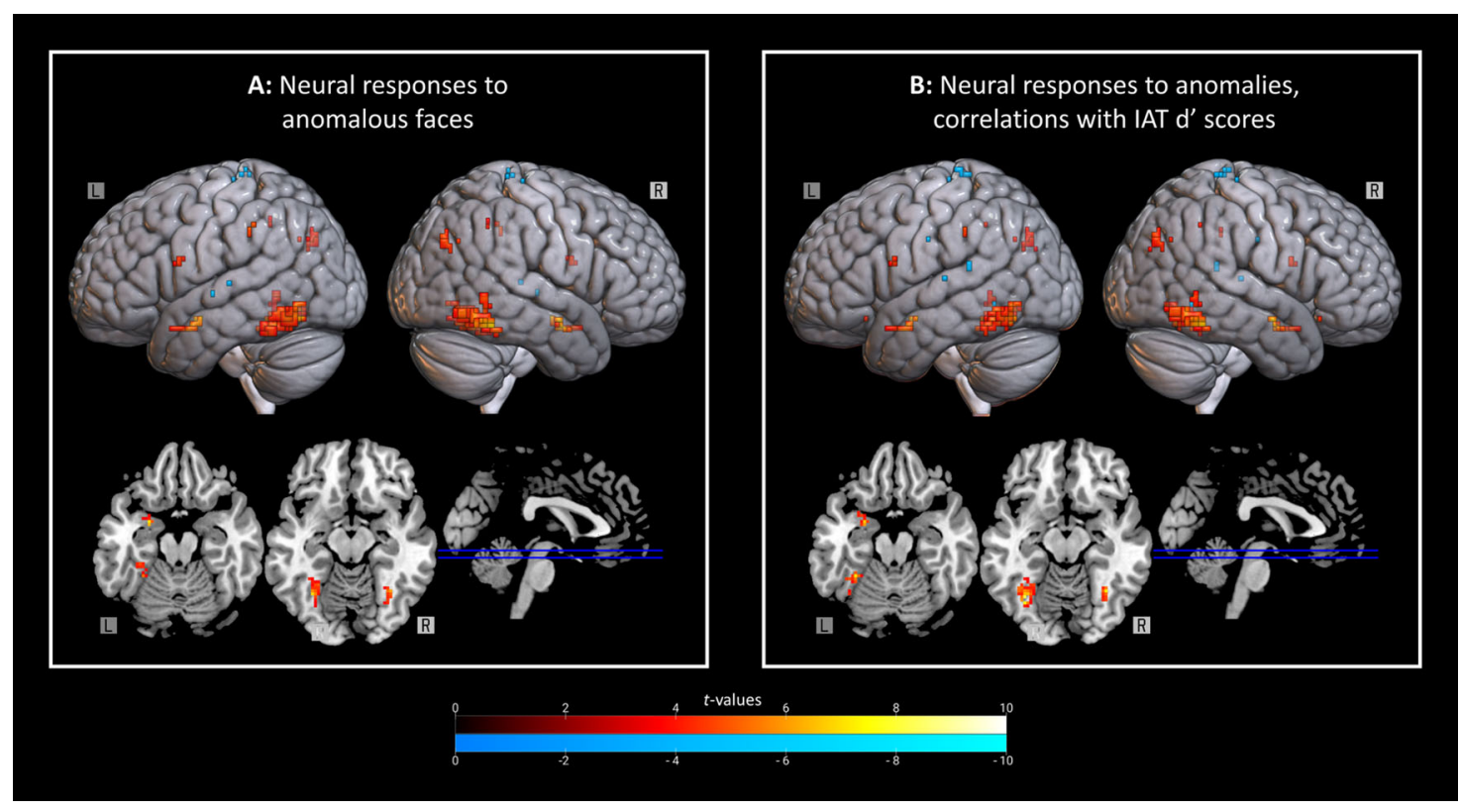

Figure 4. Selective neural responses to anomalous faces and relations to implicit bias. (A) Regions where anomalous faces elicited greater activation than beautiful and average-looking novel faces (top; no masking). Only disgusting animals and facial beauty conjointly implicated the left amygdala. Warm colors reflect increased activation for anomalous versus novel beautiful and averagelooking faces. Cool colors represent decreased activation. (B) Regions sensitive to both facial anomalies and implicit biases toward them (top; no masking). This analysis was repeated with inclusive masking for facial beauty and disgust toward animals and exclusive masking for visual salience and emotional arousal, with significant positive correlations restricted to the bilateral fusiform and left amygdala (bottom; selective masking). Warm colors reflect increased activation in response to anomalous (compared with novel beautiful and average-looking) faces-in addition, however, these clusters were further positively correlated with implicit biases. Cool colors indicate negative correlations. IAT, Implicit Association Test.

beautiful faces. It is surprising that antisociality was not more strongly affected owing to the simultaneous operation of both the "beauty-is-good" and "anomalous-is-bad" stereotypes. These findings support the view that evolutionary pressures bear more on the "anomalous-is-bad stereotype," which is further corroborated by the detection of exclusively negative relations between attractiveness and neural response amplitudes.

Our experiment enabled the disambiguation of neural responses to visually salient faces, to emotionally arousing faces, and-specifically-to anomalous faces. The visual salience response captured key nodes of the salience network, ${ }^{44}$ including the anterior insula, dACC, VS, fusiform, lateral prefrontal cortices, and posterior cingulate. The emotional arousal response implicated the fusiform, precentral, and posterior cingulate cortices, consistent with early fMRI evidence for sensitivity to emotional arousal in the fusiform. ${ }^{45,46}$ Facial anomalies elicited activation in a fronto- temporo-parietal network, with peaks in the lateral OFC, fusiform, and amygdala. Specific neural responses to facial anomalies, however-sensitive to beauty and disgust but not salience or arousalwere restricted to the fusiform and amygdala.

The amygdala and fusiform gyri demonstrated specific neural responses to anomalous facessensitive to moral emotion (i.e., disgust) and beauty (i.e., facial unattractiveness) that were independent of neural responses to salience and arousal. Disgusting animals elicited activation in the amygdala, fusiform, and precuneus. Disgusting bodily injuries implicated the fusiform and precuneus, but not the amygdala. Neither disgusting animals nor injuries elicited activation in anterior insula, as reported previously. ${ }^{47}$ Amygdala responses to disgusting stimuli have, however, been reported absent effects in the anterior insula. ${ }^{48}$ Brain responses to facial unattractiveness included the same amygdala region activated by disgusting animals (but not injuries) and fusiform areas 
sensitive to both kinds of disgusting images. Three clusters demonstrated specificity-the left fusiform, right fusiform, and left amygdala. Activations in all three tracked implicit biases, suggesting selective neural responses to anomalous faces underpin implicit biases against them. Diminishing facial beauty and disgusting animals alone, however, conjointly implicated the left amygdala.

The amygdala has been associated with feeling bad when facing tough moral choices. ${ }^{49}$ For anomalous (relative to typical) faces, parameter estimates from an ROI in the left amygdala were positively related to beliefs about justice toward others and negatively related to empathic concern. Since this region was sensitive to disgusting animals at known risk of harboring infectious diseases, we might have anticipated amygdala activation to track sensitivity to pathogen-related disgust. Together with the fusiform gyri, however, the left amygdala correlated negatively with prosociality toward anomalous faces. This finding is perhaps surprising since the amygdala and fusiform gyri were previously implicated in processing antisocial emotions (e.g., disgust), whereas prosocial emotions (e.g., empathic concern) recruit frontomesolimbic regions (e.g., subgenual cingulate cortex, sgACC). ${ }^{16}$ Moreover, larger selective amygdala responses to anomalous faces were also linked to weaker dispositional empathic concern and to stronger just-world beliefs about others.

We propose that the left amygdala integrates face perception with moral emotions (e.g., empathy) and social values (e.g., justice) to guide behaviorless emotional empathy and greater belief that the world is just together facilitate dehumanizing people with facial anomalies. The lateralization of our findings to the left amygdala is consistent with evidence that the left amygdala, specifically, is implicated in representing subjective value, ${ }^{50}$ in empathy, ${ }^{51}$ and in sociomoral cognition more broadly. ${ }^{52}$ The amygdala shares connections with frontomesolimbic regions implicated in prosocial emotions (e.g., guilt and empathy). ${ }^{16,53,54}$ Previous work has linked empathic concern to sgACC representations of guilt $t^{55}$ and dysfunctional sgACC-amygdala connectivity to pathological guilt. ${ }^{56}$ Conversely, fronto-amygdala functioning is impaired in psychopaths with deficits in empathy ${ }^{17}$ and tracks moral beliefs about the acceptability of intergroup violence. ${ }^{57}$ We suggest that the left amygdala encodes evaluations of prospective social partners informed by their facial characteristics through reciprocal connections to frontomesolimbic regions involved in empathy and valuation. ${ }^{16,58}$ Here, the sgACC is sensitive to visual salience. Fronto-amygdala connections may tether specific neural activity elicited by anomalies to general responses related to processing, for example, salient stimuli. This perspective coheres with evidence the amygdala are hubs ${ }^{59}$ that integrate aversive emotion ${ }^{60}$ with representations of social value. ${ }^{61,62}$

Several parallels between our findings and those reported in the extant literature on intergroup biases deserve attention. Meta-analytic evidence has implicated both the fusiform and amygdala in other-race categorization, ${ }^{63}$ with theoretical work underscoring the importance of visual attention to faces for social categorization and intergroup bias. ${ }^{64}$ Furthermore, the amygdala is implicated both in empathic functioning and race-based biases. ${ }^{65}$ We also find support for the proposal that dehumanization facilitates the "anomalousis-bad" stereotype. ${ }^{2,14,15}$ Masking neural responses to anomalous faces with responses to disgusting animals (but not bodily injuries) captured the left amygdala cluster from Table 1 . This pattern suggests the left amygdala is preferentially activated not by disgust toward human bodies but by disgust toward nonhuman animals. Our results, then, are consistent with a mechanistic account of dehumanization whereby dehumanized others appear human despite having subhuman essences. The results further indicate that anomalous faces elicit nonspecific dehumanizing responses that do not differentiate between specific anomalous facial features. Consistent with this interpretation, a stronger belief that the world is just for others and a disinclination to experiencing affective empathy modulated amygdala activity. These findings support theorizing that suggests the capacity to dehumanize others is inversely related to empathic functioning. ${ }^{14}$

Several limitations to the current research necessitate discussion. Owing to the heterogeneity in the etiologies of the facial anomalies-which included mostly scars but also carcinomas, swellings and growths, and facial paralysis-we could not disentangle whether specific anomalies are especially prone to triggering the "anomalous-is-bad" stereotype. We present evidence, however, that dehumanizing responses fail to differentiate between specific 
anomalies. It also bears noting that the anomalous faces were older than the nonanomalous faces. It is well established that older faces are judged less attractive than younger faces, ${ }^{66}$ raising the possibility that age may have contributed to the reported effects related to unattractiveness. Many of the bestknown databases of standardized face photographs comprise almost exclusively younger adults and feature only limited racial and/or ethnic diversity. ${ }^{28}$ Ultimately, the Chicago Face Database provided the best available match in demographic characteristics to the ChatLab Facial Anomaly Database. Similar concerns could be raised about the potential for systematic differences in the physical properties of images selected from the ChatLab Facial Anomaly Database relative to the Chicago Face Database (e.g., brightness). Some of the physical differences likely to distinguish the databases, however, reflect features that may actually be constitutive of the anomalies. The reddish discolorations in "portwine stains," for instance, are central to what makes them "anomalous." Although not possible here, the potential confounds identified above warrant additional exploration in future studies.

\section{Conclusion}

This research confirmed earlier reports that people with anomalous faces are imbued with negative personality characteristics, detected explicit biases against people with facial anomalies, and described a behavioral manifestation of the "anomalous-isbad" stereotype affecting prosociality. We suggest that dehumanization is underpinned by a suite of negative attitudes (IAT and EBQ), social cognitive biases (just-world beliefs), emotional dispositions (affective empathy), and undesirable behaviors (less generosity in the DG) - all factors associated with the functioning of the left amygdala. Understanding the psychology of the "anomalous-is-bad" stereotype may inform interventions to educate the public about the social burdens shouldered by people who look different.

\section{Acknowledgments}

This research was supported by the Penn Center for Human Appearance (awarded to A.C.) and the National Institute of Dental \& Craniofacial Research of the National Institutes of Health (F32DE029407 awarded to C.I.W.). The content is solely the responsibility of the authors and does not neces- sarily represent the official views of the National Institutes of Health. We thank Kenji Kobayashi for guidance on analyzing the economic game data.

\section{Author contributions}

All authors contributed to the study design. Data collection was performed by C.I.W. and F.H. Analyses were performed by C.I.W. and S.H. The manuscript was drafted by C.I.W. All authors provided critical revisions and approved the final version of the manuscript.

\section{Supporting information}

Additional supporting information may be found in the online version of this article.

Figure S1. Responses to the online survey of character judgments about people with and without facial anomalies.

Figure S2. Prosocial and antisocial behavior toward people with and without facial anomalies.

Table S1. The minimum number of ratings per photograph required for favorable reliability and statistical power when comparing anomalous with typical faces.

Table S2. The Explicit Bias Questionnaire (EBQ).

Table S3. Demographic characteristics corresponding to the experimental stimuli used in the fMRI and Ultimatum Game (anomalous and beautiful faces only) tasks.

Table S4. Demographic characteristics of the people whose photographs were used as experimental stimuli in the Dictator Game.

Table S5. Inferences about and responses to faces with visible anomalies, beautiful faces, and averagelooking faces (learned and novel).

Table S6. Fixed effects from the linear mixed models constructed for the Dictator and Ultimatum Games.

Table S7. Neural sensitivity to visual salience.

Table S8. Neural sensitivity to emotional arousal.

Table S9. Neural sensitivity to disgust.

Table S10. Neural sensitivity to facial beauty.

Table S11. Neural sensitivity to facial anomalies. 
Table S12. Neural responses to facial anomalies in regions independently implicated in processing disgust and facial beauty.

Table S13. Correlations between implicit biases and neural responses to facial anomalies.

\section{Competing interests}

The authors declare no competing interests.

\section{References}

1. Croley, J.A., V. Reese \& R.F. Wagner. 2017. Dermatologic features of classic movie villains. JAMA Dermatol. 153: 559564.

2. Hartung, F., A. Jamrozik, M.E. Rosen, et al. 2019. Behavioural and neural responses to facial disfigurement. Sci. Rep. 9: 8021.

3. Houston, V. \& R. Bull. 1994. Do people avoid sitting next to someone who is facially disfigured? Eur. J. Soc. Psychol. 24: 279-284.

4. Jamrozik, A., M. Oraa Ali, D.B. Sarwer, et al. 2019. More than skin deep: judgments of individuals with facial disfigurement. Psychol. Aesthetics Creat. Arts 13: 117-129.

5. Madera, J.M. \& M.R. Hebl. 2012. Discrimination against facially stigmatized applicants in interviews: an eye-tracking and face-to-face investigation. J. Appl. Psychol. 97: 317-330.

6. Mojon-Azzi, S.M., W. Potnik \& D.S. Mojon. 2008. Opinions of dating agents about strabismic subjects' ability to find a partner. Br. J. Ophthalmol. 92: 765-769.

7. Dion, K., E. Berscheid \& E. Walster. 1972. What is beautiful is good. J. Pers. Soc. Psychol. 24: 285-290.

8. Eagly, A.H., R.D. Ashmore, M.G. Makhijani, et al. 1991. What is beautiful is good, but ...: a meta-analytic review of research on the physical attractiveness stereotype. Psychol. Bull. 110: 109-128.

9. Rhodes, G. 2006. The evolutionary psychology of facial beauty. Annu. Rev. Psychol. 57: 199-226.

10. Bzdok, D., R. Langner, S. Caspers, et al. 2011. ALE metaanalysis on facial judgments of trustworthiness and attractiveness. Brain Struct. Funct. 215: 209-223.

11. Griffin, A.M. \& J.H. Langlois. 2006. Stereotype directionality and attractiveness stereotyping: is beauty good or is ugly bad? Soc. Cogn. 24: 187-206.

12. Zebrowitz, L.A. \& J.M. Montepare. 2008. Social psychological face perception: why appearance matters. Soc. Personal. Psychol. Compass 2: 1497-1517.

13. Harris, L.T. \& S.T. Fiske. 2006. Dehumanizing the lowest of the low. Psychol. Sci. 17: 847-853.

14. Haslam, N. \& S. Loughnan. 2014. Dehumanization and infrahumanization. Annu. Rev. Psychol. 65: 399-423.

15. Over, H. 2021. Seven challenges for the dehumanisation hypothesis. Perspect. Psychol. Sci. 16: 3-13.

16. Zahn, R., R. de Oliveira-Souza \& J. Moll. 2020. Moral motivation and the basal forebrain. Neurosci. Biobehav. Rev. 108: 207-217.

17. Decety, J., R. Pape \& C.I. Workman. 2018. A multilevel social neuroscience perspective on radicalization and terrorism. Soc. Neurosci. 13: 511-529.
18. Ryan, S., M. Oaten, R.J. Stevenson, et al. 2012. Facial disfigurement is treated like an infectious disease. Evol. Hum. Behav. 33: 639-646.

19. Shanmugarajah, K., S. Gaind, A. Clarke, et al. 2012. The role of disgust emotions in the observer response to facial disfigurement. Body Image 9: 455-461.

20. Tybur, J.M., D. Lieberman, R. Kurzban, et al. 2013. Disgust: evolved function and structure. Psychol. Rev. 120: 65-84.

21. Landy, J.F. \& G.P. Goodwin. 2015. Does incidental disgust amplify moral judgment? A meta-analytic review of experimental evidence. Perspect. Psychol. Sci. 10: 518-536.

22. Squires, N.K., K.C. Squires \& S.A. Hillyard. 1975. Two varieties of long-latency positive waves evoked by unpredictable auditory stimuli in man. Electroencephalogr. Clin. Neurophysiol. 38: 387-401.

23. Smeets, P., R. Bauer \& U. Gneezy. 2015. Giving behavior of millionaires. Proc. Natl. Acad. Sci. USA 112: 1064110644.

24. Korndörfer, M., B. Egloff \& S.C. Schmukle. 2015. A large scale test of the effect of social class on prosocial behavior. PLoS One 10: e133193.

25. Curran, P.G. 2016. Methods for the detection of carelessly invalid responses in survey data. J. Exp. Soc. Psychol. 66: 419.

26. Yarkoni, T. 2009. Big correlations in little studies: inflated fMRI correlations reflect low statistical powercommentary on Vul et al. (2009). Perspect. Psychol. Sci. 4: 294-298.

27. Geuter, S., G. Qi, R.C. Welsh, et al. 2018. Effect size and power in fMRI group analysis. https://doi.org/10.1101/ 295048.

28. Workman, C.I. \& A. Chatterjee. 2020. The Face Image MetaDatabase (fIMDb) \& ChatLab Facial Anomaly Database (CFAD): tools for research on face perception and social stigma. https://doi.org/10.31234/osf.io/54utr.

29. Ma, D.S., J. Correll \& B. Wittenbrink. 2015. The Chicago Face Database: a free stimulus set of faces and norming data. Behav. Res. Methods 47: 1122-1135.

30. Bradley, M.M. \& P.J. Lang. 1994. Measuring emotion: the self-assessment manikin and the semantic differential. J. Behav. Ther. Exp. Psychiatry 25: 49-59.

31. Lucas, T., L. Zhdanova \& S. Alexander. 2011. Procedural and distributive justice beliefs for self and others. J. Individ. Differ. 32: 14-25.

32. Davis, M.H. 1983. Measuring individual differences in empathy: evidence for a multidimensional approach. J. Pers. Soc. Psychol. 44: 113-126.

33. Tybur, J.M., D. Lieberman \& V. Griskevicius. 2009. Microbes, mating, and morality: individual differences in three functional domains of disgust. J. Pers. Soc. Psychol. 97: $103-122$.

34. Kurdi, B., T.C. Mann, T.E.S. Charlesworth, et al. 2019. The relationship between implicit intergroup attitudes and beliefs. Proc. Natl. Acad. Sci. USA 116: 5862-5871.

35. Greenwald, A.G., T.A. Poehlman, E.L. Uhlmann, et al. 2009. Understanding and using the Implicit Association Test: III. Meta-analysis of predictive validity. J. Pers. Soc. Psychol. 97: $17-41$.

36. Kahneman, D., J.L. Knetsch \& R.H. Thaler. 1986. Fairness and the assumptions of economics. J. Bus. 59. S285. 
37. Güth, W., R. Schmittberger \& B. Schwarze. 1982. An experimental analysis of ultimatum bargaining. J. Econ. Behav. Organ. 3: 367-388.

38. Knolle, F., A.O. Ermakova, A. Justicia, et al. 2018. Brain responses to different types of salience in antipsychotic naive first episode psychosis: an fMRI study. Transl. Psychiatry 8: 196.

39. Warbrick, T., M. Reske \& N.J. Shah. 2013. Do EEG paradigms work in fMRI? Varying task demands in the visual oddball paradigm: implications for task design and results interpretation. Neuroimage 77: 177-185.

40. Clark, V.P., S. Fannon, S. Lai, et al. 2001. Paradigmdependent modulation of event-related fMRI activity evoked by the oddball task. Hum. Brain Mapp. 14: 116-127.

41. Haberkamp, A., J.A. Glombiewski, F. Schmidt, et al. 2017. The DIsgust-RelaTed-Images (DIRTI) database: validation of a novel standardized set of disgust pictures. Behav. Res. Ther. 89: 86-94.

42. Dan-Glauser, E.S. \& K.R. Scherer. 2011. The Geneva Affective Picture Database (GAPED): a new 730-picture database focusing on valence and normative significance. Behav. Res. Methods 43: 468-477.

43. Lane, T. 2016. Discrimination in the laboratory: a metaanalysis of economics experiments. Eur. Econ. Rev. 90: 375402.

44. Seeley, W.W., V. Menon, A.F. Schatzberg, et al. 2007. Dissociable intrinsic connectivity networks for salience processing and executive control. J. Neurosci. 27: 2349-2356.

45. Lang, P.J., M.M. Bradley, J.R. Fitzsimmons, et al. 1998. Emotional arousal and activation of the visual cortex: an fMRI analysis. Psychophysiology 35: 199-210.

46. Chatterjee, A., A. Thomas, S.E. Smith, et al. 2009. The neural response to facial attractiveness. Neuropsychology 23: 135143.

47. Calder, A.J., A.D. Lawrence \& A.W. Young. 2001. Neuropsychology of fear and loathing. Nat. Rev. Neurosci. 2: 352-363.

48. Schienle, A., A. Schäfer, A. Hermann, et al. 2006. fMRI responses to pictures of mutilation and contamination. $\mathrm{Neu}$ rosci. Lett. 393: 174-178.

49. May, J., C.I. Workman, J. Haas, et al. 2021. The neuroscience of moral judgment: empirical and philosophical developments. In Neuroscience and Philosophy. Bringing the Fields Together. W. Sinnott-Armstrong \& F. De Brigard, Eds.: 1-21. Cambridge, MA: MIT Press.

50. Mormann, F., M. Bausch, S. Knieling, et al. 2019. Neurons in the human left amygdala automatically encode subjective value irrespective of task. Cereb. Cortex 29: 265-272.

51. Del Casale, A., G.D. Kotzalidis, C. Rapinesi, et al. 2017. Neural functional correlates of empathic face processing. Neurosci. Lett. 655: 68-75.
52. Eres, R., W.R. Louis \& P. Molenberghs. 2018. Common and distinct neural networks involved in fMRI studies investigating morality: an ALE meta-analysis. Soc. Neurosci. 13: 384398.

53. Alheid, G.F. 2006. Extended amygdala and basal forebrain. Ann. N.Y. Acad. Sci. 985: 185-205.

54. Ongür, D., J.L. Price \& D. Ongur. 2000. The organization of networks within the orbital and medial prefrontal cortex of rats, monkeys and humans. Cereb. Cortex 10: 206-219.

55. Zahn, R., R. de Oliveira-Souza, I. Bramati, et al. 2009. Subgenual cingulate activity reflects individual differences in empathic concern. Neurosci. Lett. 457: 107-110.

56. Workman, C.I., K.E. Lythe, S. McKie, et al. 2016. Subgenual cingulate-amygdala functional disconnection and vulnerability to melancholic depression. Neuropsychopharmacology 41: 2082-2090.

57. Workman, C.I., K.J. Yoder \& J. Decety. 2020. The dark side of morality: neural mechanisms underpinning moral convictions and judgments about violence. AJOB Neurosci. 11: 269-284.

58. Bartra, O., J.T. McGuire \& J.W. Kable. 2013. The valuation system: a coordinate-based meta-analysis of BOLD fMRI experiments examining neural correlates of subjective value. Neuroimage 76: 412-427.

59. Adolfi, F., B. Couto, F. Richter, et al. 2017. Convergence of interoception, emotion, and social cognition: a twofold fMRI meta-analysis and lesion approach. Cortex 88: 124142.

60. Todorov, A. \& A.D. Engell. 2008. The role of the amygdala in implicit evaluation of emotionally neutral faces. Soc. Cogn. Affect. Neurosci. 3: 303-312.

61. Rosenberger, L.A., C. Eisenegger, M. Naef, et al. 2019. The human basolateral amygdala is indispensable for social experiential learning. Curr. Biol. 29: 3532-3537.e3.

62. Liang, X., L.A. Zebrowitz \& Y. Zhang. 2010. Neural activation in the "reward circuit" shows a nonlinear response to facial attractiveness. Soc. Neurosci. 5: 320-334.

63. Bagnis, A., A. Celeghin, M. Diano, et al. 2020. Functional neuroanatomy of racial categorization from visual perception: a meta-analytic study. Neuroimage 217: 116939.

64. Bagnis, A., A. Celeghin, C.O. Mosso, et al. 2019. Toward an integrative science of social vision in intergroup bias. Neurosci. Biobehav. Rev. 102: 318-326.

65. Han, S. 2018. Neurocognitive basis of racial ingroup bias in empathy. Trends Cogn. Sci. 22: 400-421.

66. Foos, P.W. \& M.C. Clark. 2011. Adult age and gender differences in perceptions of facial attractiveness: beauty is in the eye of the older beholder. J. Genet. Psychol. 172: 162-175. 